The Astrophysical Journal, 183: 1005-1023, 1973 August 1

(C) 1973. The American Astronomical Society. All rights reserved. Printed in U.S.A.

\title{
PHOTOSPHERIC FACULAE AND THE SOLAR OBLATENESS: A REPLY TO "FACULAE AND THE SOLAR OBLATENESS" BY R. H. DICKE
}

Gary A. Chapman

San Fernando Observatory, Space Physics Laboratory, The Aerospace Corporation AND

ANDREW P. INGERSOLL

Division of Geological and Planetary Sciences California Institute of Technology Received 1972 July 19; revised 1973 February 14

\section{ABSTRACT}

Dicke has recently contested our statement in an earlier paper that faculae could account for a large part, if not all, of the solar oblateness signal measured by Dicke and Goldenberg in 1966. Using the facular oblateness signal published in our earlier paper and some hitherto unpublished data from his 1966 observations, he concludes that faculae account for only a small part (11 percent) of the observed excess oblateness. His analysis considers data only from a restricted 48-day sample and is based on the assumption that only the observed oblateness signal is subject to error.

Our analysis considers data from all 64 days on which observations were made, and is based on the assumption that both the observed oblateness signal and the facular signal are subject to error. We find that faculae account for at least one-third to one-half of the observed excess oblateness, depending on whether 48 days or 64 days are used in the analysis. Moreover, faculae may account for all of the observed excess oblateness provided the facular error is sufficiently large. Thus faculae cannot be excluded as the major source of Dicke and Goldenberg's 1966 oblateness signal. Subject headings: faculae, solar — rotation, solar — gravitation

\section{INTRODUCTION}

In a recent paper (Chapman and Ingersoll 1972) we found that a sizeable portion of the solar oblateness signal measured at Princeton (Dicke and Goldenberg 1967) was probably the result of photospheric faculae. That conclusion was based on several approaches. First, observations of facular limb darkening indicate that faculae have the necessary dependence on viewing angle to contribute to Dicke and Goldenberg's oblateness signal (Ingersoll and Spiegel 1971). Second, published estimates of the areal coverage of faculae suggest that faculae are sufficiently widespread on the solar surface to account for the observed oblateness signal. Third, there appears to be a significant correlation between the signal measured at Princeton and our own "facular oblateness signal" determined from photographs taken daily at the San Fernando Observatory in 1966.

Of these three approaches, the third is potentially the most powerful. However, in our study we were limited because Dicke and Goldenberg's 1966 oblateness signal was not available for each day of observation. Thus our statistical analysis was based on nine points from figure 3 of Dicke $(1970 b)$, each point representing the average signal for an approximately two-week period.

Following our paper, Dicke (1972a) published a statistical analysis of faculae using the hitherto unpublished daily signals from his 1966 observations (fig. 1 of Dicke 1972a; and Dicke 1972b), which he compared with the daily facular signals from our earlier paper (table 1 of Chapman and Ingersoll 1972). He concluded that faculae 
account for only about 11 percent of the excess oblateness signal (the signal corrected for solar surface rotation), or about 9 percent of the total oblateness signal. The present paper is a reply to Dicke, and is based on the same two sets of data that Dicke used in his analysis. We find that the facular contribution is considerably greater than Dicke's 11 percent estimate, and may be as large as 100 percent of the excess oblateness signal.

One of the main differences between our analysis and Dicke's is that ours is based on the assumption that both the measured oblateness signal and the facular signal are subject to error, whereas Dicke's is based on the assumption that only the measured oblateness signal is subject to error. Since the ratio of errors of the two signals is largely unknown, a precise estimate of the facular contribution cannot be made. However, we can demonstrate the existence of a statistical relationship between the two signals with high confidence, whatever the value of the error ratio. Furthermore, we can establish lower and upper bounds on the percentage contribution of faculae to Dicke and Goldenberg's signal. These bounds are approximately 17 percent and 100 percent, respectively. The former follows if we assume that the ratio $\epsilon$ of errors in the facular signal to those in the oblateness signal is small; the latter follows if we assume that this ratio is large. Using a conservative estimate of $\epsilon$, we find that faculae contribute at least 35 percent of the excess signal (see $\S$ III $a$ ).

The paper is organized in the following manner. In $\S$ II we present our statistical analysis of the data, treating the error ratio $\epsilon$ as an unknown parameter. We use a generalized method of least squares, useful when both variables are subject to error. This method is described fully in Appendix A. In $\S$ III we discuss sources of error, and present several different estimates of the error ratio $\epsilon$. The arguments in this section are necessarily qualitative, and are themselves subject to error. In $\S$ IV we review the evidence for and against faculae as a source of Dicke and Goldenberg's excess signal and conclude that faculae certainly account for an appreciable part of the signal and may account for all of it.

In spite of the uncertainties in our conclusions, we feel that our work is important. Recall that the existence of a true gravitational oblateness, as implied by Dicke and Goldenberg's data, would upset the apparent agreement between the observed value of Mercury's excess perihelion shift and Einstein's predicted value. Thus if the Sun is oblate by the amount claimed by Dicke, the fact that general relativity accounts for the observed perihelion shift must be the result of coincidence. Our work removes the obligation to accept this coincidence. We have shown, in spite of the uncertainties in comparing our facular signal with the observed signal, that faculae offer an acceptable alternate explanation of Dicke and Goldenberg's excess solar oblateness.

\section{STATISTICAL ANALYSIS}

Our aim is to determine the relative contribution of faculae to Dicke and Goldenberg's excess oblateness signal. For each day of observation we are given the following three quantities: $\sin 2 P_{i}$, or $\left(\Delta r_{d} / \Delta r\right)$ in Dicke's notation, where $P_{i}$ is the angle on the solar disk between the solar rotation vector and geographic north; $O_{i}$, or $(\Delta r / r)_{d}$ in Dicke's notation, the daily oblateness signal from Dicke $(1972 a, b)$; and $F_{i}$, the daily facular signal from table 1 of Chapman and Ingersoll (1972). The subscript $i$ denotes the day of observation. As shown in our earlier paper, the ensemble means of $F_{i}$ and $O_{i}$ are each equal to a constant times $\sin 2 P_{i}$. We wish to estimate the ratio of these two constants, and thereby obtain an estimate of the fractional contribution of faculae to Dicke and Goldenberg's signal. However, since the magnitude of $F_{i}$ is largely unknown, the problem is to determine the ratio of these two constants simply from the form of the functions $O$ and $F$, that is, from their relative variations from day to day. 
Values of $F_{i}$ exist for 135 consecutive calendar days in 1966; values of $O_{i}$ exist only for 64 days in the same period. These 64 days are not always consecutive. Dicke $(1972 a)$ considers the oblateness data $O_{i}$ from 16 days at the beginning and end of the 1966 observing period to be less reliable, and has based his most recent analyses on a 48-day sample. In what follows, we will present results for both the 48-day sample and the 64-day sample.

\section{a) The Relation between $O$ and $F$}

Our results cannot depend on the absolute magnitudes of the variables $O$ and $F$. Therefore, $O$ and $F$ have been scaled to $\sin 2 P$ such that the optimum values of the coefficients $C_{1}$ and $C_{2}$ which minimize the sums

$$
\begin{aligned}
& \sum_{i}\left(O_{i}-C_{1} \sin 2 P_{i}-C_{3}\right)^{2}, \\
& \sum_{i}\left(F_{i}-C_{2} \sin 2 P_{i}-C_{4}\right)^{2},
\end{aligned}
$$

are both equal to unity. This scaling to $\sin 2 P$ is done separately for the 48-day and the 64-day samples, although the differences in the scaling factors for the two samples are small. We then define residuals from $\sin 2 P$ as follows:

$$
\delta O_{i}=O_{i}-C_{1} \sin 2 P_{i}-C_{3}, \quad \delta F_{i}=F_{i}-C_{2} \sin 2 P_{i}-C_{4} .
$$

The sums of residuals $\sum_{i} \delta O_{i}$ and $\sum_{i} \delta F_{i}$ are identically zero, which follows from the above definitions. In all cases studied, the values of the constant terms $C_{3}$ and $C_{4}$ are small and not statistically significant.

Both $O$ and $F$ are subject to error, and therefore the observations $O_{i}$ and $F_{i}$ differ from the "true" values by amounts which we shall call the "errors" of $O_{i}$ and $F_{i}$. The variances, or mean square errors (ensemble mean), are assumed to be the same for each day of observation, and are denoted by $\sigma_{O}^{2}$ and ${\sigma_{F}}^{2}$, respectively. We define the error ratio $\epsilon$ as follows:

$$
\epsilon=\sigma_{F} / \sigma_{O}
$$

The value of $\epsilon$ cannot be uniquely determined from the data $O_{i}, F_{i}$, and $\sin 2 P_{i}$ alone. In $\S$ III we shall discuss various ways of estimating $\epsilon$ using additional information. In the present section the value of $\epsilon$ will be treated as unknown. Note that the errors are not the same as residuals from sin $2 P$, defined in equations (2) above.

We now assume that a linear relation exists between the variables $O, F$, and $\sin 2 P$. This relation may be written in the form

$$
O=C_{5} F+C_{6} \sin 2 P+C_{7} .
$$

The actual data $O_{i}, F_{i}$, and $\sin 2 P_{i}$ satisfy this relation only in some least-squares sense. In Appendix $A$ we describe how to determine the coefficients $C_{5}, C_{6}, C_{7}$ and their standard deviations $\sigma_{5}, \sigma_{6}, \sigma_{7}$, using a generalized method of least squares. This method is symmetric in the variables $O$ and $F$, and is valid for all values of the error ratio $\epsilon$. In the limits $\epsilon \rightarrow 0$ and $\epsilon \rightarrow \infty$ it reduces to the more familiar method of least squares, valid when only one variable is subject to error. In Appendix A it is shown that Dicke's results correspond roughly to the case $\epsilon \rightarrow 0$, valid when the only source of error is associated with the oblateness signal $O$.

Before presenting numerical results, we comment on the physical significance of the coefficients $C_{5}, C_{6}, C_{7}$. Since both $O$ and $F$ are scaled to $\sin 2 P$ as described in formula 
(1), the optimum values of the coefficients $C_{5}$ and $C_{6}$ also satisfy the relation

$$
C_{5}+C_{6}=1 \text {. }
$$

The constant term $C_{7}$ is always small and not statistically significant. Thus from equations (4) and (5) it follows that $C_{5}$ represents the fraction of Dicke and Goldenberg's total signal due to faculae, and $C_{6}$ represents the remaining fraction due to all other sources. Since 0.185 of Dicke and Goldenberg's signal is associated with solar surface rotation, the "excess" signal is only $1-0.185=1 / 1.225$ of the whole. Thus $1.225 C_{5}=C_{5}$ ' represents the fraction of Dicke and Goldenberg's excess signal due to faculae.

\section{b) Numerical Results: $C_{5}{ }^{\prime}$ versus $\epsilon$}

Values of $C_{5}{ }^{\prime}$ and their standard deviations are presented in tables 1 and 2 and in figure 1 . The numbers in table 1 were computed from equations (A7), (A8), and (A15), using the facular signal from table 1 of Chapman and Ingersoll (1972). The numbers in table 2 were computed from the same equations, using a facular signal modified as described in Appendix B to include sunspots. Values of $C_{6}$ may be computed from these numbers using equation (5). Standard deviations of $C_{6}$ are equal to those of $C_{5}$, again because of equation (5).

From tables 1 and 2 we see that if the error in the facular signal is much larger than the error in the oblateness signal (corresponding to $\epsilon \rightarrow \infty$ ), the data suggest that approximately all of the excess signal is due to faculae $\left(C_{5}{ }^{\prime}=0.934\right.$ for the 48-day sample and $C_{5}{ }^{\prime}=1.259$ for the 64-day sample, neglecting sunspots; or $C_{5}{ }^{\prime}=1.14$ and 1.34 , including sunspots). From figure 1 , we see that for $\epsilon=3$ the estimated facular contribution is over 50 percent for the 48-day sample, and essentially 100 percent for the 64-day sample. These data form the basis of our conclusion that faculae cannot be excluded as a potential source of the bulk of Dicke and Goldenberg's excess oblateness signal. In $\S$ III we shall discuss the possible values of $\epsilon$, as well as the relative merits of the 48- and 64-day samples.

\section{TABLE 1}

Facular Contribution, $C_{5}{ }^{\prime}$, to the Excess Solar OBLATENESS NEGLECTING SUNSPOTS

\begin{tabular}{lcc}
\hline & \multicolumn{2}{c}{$1.225\left(C_{5} \pm \sigma_{5}\right)=C_{5}{ }^{\prime} \pm \sigma_{5}{ }^{\prime}$} \\
\cline { 2 - 3 } & 64-Day Sample & 48-Day Sample \\
\hline$\epsilon \rightarrow 0 \ldots \ldots \ldots$ & $0.313 \pm 0.069$ & $0.140 \pm 0.049$ \\
$\epsilon \rightarrow \infty \ldots \ldots$ & $1.259 \pm 0.278$ & $0.933 \pm 0.33$ \\
\hline
\end{tabular}

TABLE 2

Facular Contribution, $C_{5}{ }^{\prime}$, to the Excess Solar OblateNESS INCLUDING THE EFFECT OF SUNSPOTS

\begin{tabular}{lrr}
\hline \hline & \multicolumn{2}{c}{$1.225\left(C_{5} \pm \sigma_{5}\right)=C_{5}{ }^{\prime} \pm \sigma_{5}{ }^{\prime}$} \\
\cline { 2 - 3 } & 64-Day Sample & 48-Day Sample \\
\hline$\epsilon \rightarrow 0 \ldots \ldots \ldots$ & $0.349 \pm 0.075$ & $0.167 \pm 0.060$ \\
$\epsilon \rightarrow \infty \ldots \ldots \ldots$ & $1.34 \pm 0.286$ & $1.147 \pm 0.410$ \\
\hline
\end{tabular}




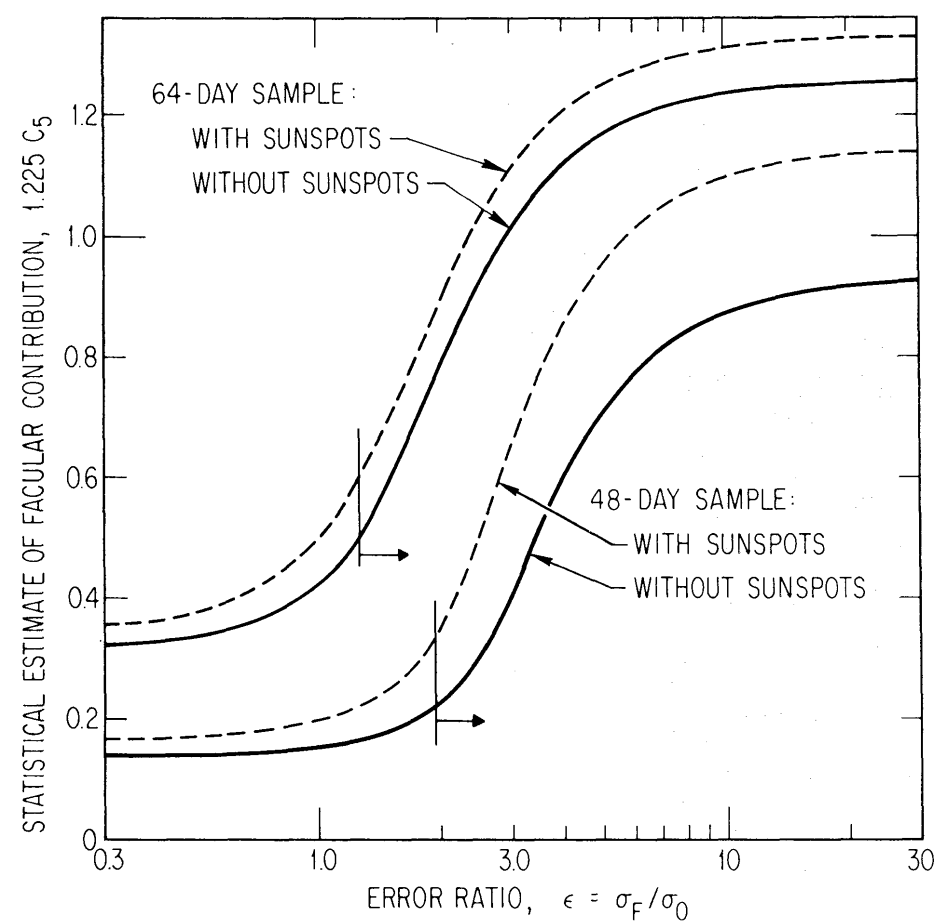

FIG. 1.-Estimates of the facular contribution to Dicke and Goldenberg's excess oblateness signal. Excess signal is that part which is not due to solar surface rotation, and $1.225 C_{5}$ is the fraction of this excess signal which is due to faculae. Estimates of $1.225 C_{5}$ depend on the assumed value of $\epsilon$, the ratio of the error in the facular signal $F$ to the error in the oblateness signal $O$. The dashed curves include sunspots; the solid curves do not. The 64- and 48-day curves refer to the same days of observation, with the exception of 16 days considered less reliable by Dicke $(1972 a)$. However, as discussed in the text, the 64-day sample may provide the best estimate of $1.225 C_{5}$. Our most objective and conservative estimates of $\epsilon$ are 1.28 and 1.97 for the 64- and 48-day samples, respectively, and are denoted by vertical lines in the figure. The corresponding estimates of $1.225 C_{5}$ from the dashed curve are 0.61 and 0.35 , respectively.

Dicke's result, that faculae represent an 11 percent contribution to the excess oblateness signal, corresponds to the number $0.140(\epsilon \rightarrow 0$, 48-day sample) appearing in table 1. The difference between these two numbers $(0.11$ and 0.140$)$ is due to two factors: first, Dicke uses $F$ rather than $\delta F$ in his regression analysis; second, Dicke defines residuals from $\sin 2 P$ without including the constant terms $C_{3}$ and $C_{4}$ which appear in expressions (1) and (2). In any case we see from figure 1 that the limit $\epsilon \rightarrow 0$ merely gives a lower bound estimate of $C_{5}{ }^{\prime}$. To make a better estimate of $C_{5}{ }^{\prime}$ requires an estimate of $\epsilon$, which cannot be uniquely obtained from these data alone.

The standard deviations given in table 1 provide strong evidence that the relation between $O$ and $F$ is statistically significant. For the 48-day sample the value of $C_{5} / \sigma_{5}$, computed from equation (A15), is 2.8; and for the 64-day sample the value is 4.5 . The former value is significant at the 0.006 level and the latter value is significant at the $1 \times 10^{-4}$ level. As shown in Appendix A, the value of $C_{5} / \sigma_{5}$ is the same for $\epsilon \rightarrow 0$ and $\epsilon \rightarrow \infty$, and is somewhat larger for other values of $\epsilon$. These levels of significance are therefore conservative, and hold for all values of $\epsilon$. When sunspots are included, the values of $C_{5} / \sigma_{5}$ are 2.8 and 4.7 for the 48-day and 64-day samples, and the corresponding levels of significance are 0.008 and $5 \times 10^{-5}$, respectively.

The above inferences concerning the significance of the coefficient $C_{5}$ are based on the assumptions that errors of $O$ and $F$ are Gaussian and that errors on different days 


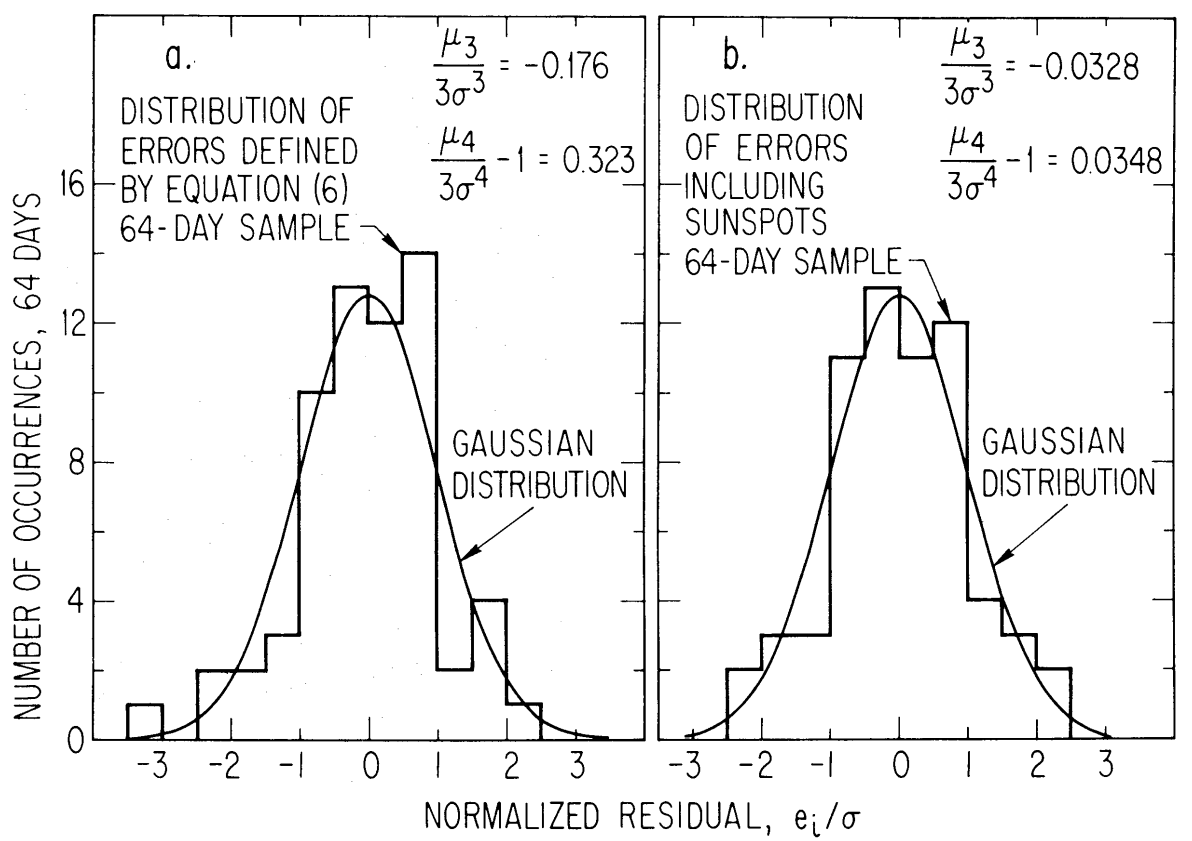

FIG. 2.-Distribution of errors defined by equation (6) for the 64-day sample: (a) sunspots not included; $(b)$ sunspots included. A $\chi^{2}$ test indicates that the non-Gaussian nature of the distribution in $(a)$ is not significant at the 40 percent level, and in $(b)$ is even less significant.

are uncorrelated. As shown in Appendix A, these assumptions may be tested by computing $e_{i}$, the overall error in equation (4):

$$
e_{i}=O_{i}-C_{5} F_{i}-C_{6} \sin 2 P_{i}-C_{7} \text {. }
$$

Errors in both $O$ and $F$ contribute to $e_{i}$ for all $\epsilon$, although errors in $O$ predominate for $\epsilon \rightarrow 0$ and errors in $F$ predominate for $\epsilon \rightarrow \infty$. Figure $2 a$ presents results for the case $\epsilon \rightarrow \infty$. (The $\epsilon \rightarrow 0$ case is not significantly different.) Here we have plotted the distribution of $e_{i} / \sigma$, where $\sigma$ is the standard deviation of the $e_{i}$. The distribution conforms to a Gaussian distribution within quite acceptable limits. If we denote the mean value of $\left(e_{i}\right)^{n}$ by $\mu_{n}$, we find $\mu_{1}=0, \mu_{2}=\sigma^{2}, \mu_{3} / 3 \sigma^{3}=-0.176$, and $\left(\mu_{4} / 3 \sigma^{4}\right)-1=$ 0.323 . When sunspots are included, the latter two quantities become -0.033 and 0.035 , respectively. The distribution of $e_{i} / \sigma$ when sunspots are included is shown in figure $2 b$. For the 48-day sample, including sunspots, we find $\mu_{3} / 3 \sigma^{3}=0.015$ and $\left(\mu_{4} / 3 \sigma^{4}\right)-1$ $=0.141$.

Further, according to a $\chi^{2}$ test, the errors $e_{i}$ are reasonably Gaussian. This test indicates that the non-Gaussian nature of $e_{i}$ is not significant at the 40 percent level without sunspots and is even less significant when sunspots are included. In short, there is little reason to reject the assumption that errors in $O$ and $F$ have a Gaussian distribution.

We emphasize that neither $O$ nor $F$, nor their residuals from $\sin 2 P, \delta O$ and $\delta F$, must be Gaussian in order that the above conclusions be valid. Fluctuations in $O, F$, $\delta O$, and $\delta F$ are partly associated with intrinsic solar fluctuations, and these may or may not lead to a Gaussian distribution. On the other hand, we assume that the linear relation (4) removes this dependence on intrinsic solar fluctuations, and that the remaining errors are Gaussian. This is the hypothesis which we tested above and in figure 2 , and which must be satisfied if the value of $C_{5} / \sigma_{5}$ is to be used as a Gaussian measure of statistical significance.

We also require $e_{i}$ to be uncorrelated on successive days. Figure 4 (bottom) shows 
the autocorrelation of $e_{i}$ for $\epsilon \rightarrow \infty$. For the 34 pairs of observations $\left(e_{i}\right.$ versus $\left.e_{i+1}\right)$ the correlation coefficient is 0.02 (for $\epsilon \rightarrow \infty$ ), which is not significant. (For $\epsilon \rightarrow 0$ this correlation coefficient is 0.21 , which is significant at about the 35 percent level.) The correlation coefficients $\left(\delta O_{i}\right.$ versus $\left.\delta O_{i+1}\right)$ and $\left(\delta F_{i}\right.$ versus $\left.\delta F_{i+1}\right)$ are 0.58 and 0.31 , respectively, the first of which is significant at the 5 percent level and the second significant at the 10 percent level. Thus, although the residuals from $\sin 2 P$ show a significant autocorrelation for a 1-day lag, the error $e_{i}$ does not show this. Thus we find no evidence that errors $e_{i}$ from successive calendar days are significantly correlated. The $e_{i}$ for $\epsilon \rightarrow \infty$ are plotted in figure 6 against day number $i$ for all 64 days of observation.

\section{c) Further Numerical Results: Correlation Analyses}

We now present the results of lagged cross correlations and autocorrelations, neglecting sunspots, with lags ranging up to two solar rotations.

For two variables $\phi$ and $\psi$ we define the correlation coefficient $r_{j}(\phi, \psi)$ as follows:

$$
r_{j}(\phi, \psi)=\sum_{i}\left(\phi_{i}-\bar{\phi}\right)\left(\psi_{i+j}-\bar{\psi}\right) /\left[\sum_{i}\left(\phi_{i}-\bar{\phi}\right)^{2} \sum_{i}\left(\psi_{i+j}-\bar{\psi}\right)^{2}\right]^{1 / 2} \text {. }
$$

The sums are taken over a data set consisting of all pairs $\left(\phi_{i}, \psi_{i+j}\right)$ for which data exist. The number of pairs in the data set depends on $j$, the number of days lagged, and is denoted by $N_{j}$. The means $\bar{\phi}$ and $\bar{\psi}$ are means for the data set, and therefore depend on $j$. For $j=0$, we have $N_{j}=48$ or 64 , depending on whether the 48-day or 64-day sample is used. Moreover, for $j=0$ the above definition of $r(\delta F, \delta O)$ agrees with the definition (A14), since $\langle\delta F\rangle=\langle\delta O\rangle=0$ for the 48-day and 64-day samples. For $j \neq 0$, the means $\langle\delta F\rangle$ and $\langle\delta O\rangle$ are quite small, and are never statistically significant.

The top curve of figure 3 shows the autocorrelation coefficient $r_{j}(\delta F, \delta F)$, for all the facular data, plotted against $j$, the number of days lagged. The number of data pairs $N_{j}$ varies from 135 at $j=0$ to 99 at $j=36$. Significant correlations exist for $j=1, j=9-17$ ( $\frac{1}{2}$ solar rotation), and $j=23-30$ days ( 1 solar rotation). The greater widths of the peaks near $\frac{1}{2}$ and 1 solar rotation, in contrast to the relatively narrow peak at $j=0$, is due to gradual changes in facular areas with time due to the migration of the magnetic fields. These main peaks in the autocorrelation curve are easily explained by solar rotation. Recall that the data refer to the diagonal (NW-SE) component of solar oblateness, and that faculae typically occur in a latitude band centered at $23^{\circ} \mathrm{N}$ latitude. Thus the negative correlation at $\frac{1}{2}$ solar rotation is a consequence of the opposite contribution to the diagonal component of oblateness when a facular region reappears on the opposite limb after $\frac{1}{2}$ solar rotation. (It must always be remembered that the mere presence of faculae on the limb does not necessarily imply a positive facular contribution to the diagonal component of the excess solar oblateness.) The double peaks at $j=10$ and $j=15$ days lag can be explained by the fact that the measurement zones on the east and west solar limbs are not on a diameter and thus the rotation times between these zones are not equal. This effect will be seen clearly in the cross-correlations to be discussed shortly.

The middle and lower curves of figure 3 show the autocorrelation coefficients $r_{j}(\delta F, \delta F)$ and $r_{j}(\delta O, \delta O)$, where only data from 48-day samples have been used. The lower curve is similar to figure 2 of Dicke $(1972 c)$. The number of data pairs, $N_{j}$, for these curves is typically about 25 near the origin and about 20 near 1 solar rotation; thus each point is based on about one-fifth as many data as in the upper curve. The value of $N_{j}$ has been used to determine the 0.05 lines of significance shown on either side of the $r=0$ axis on each correlation curve.

Dicke $(1972 c)$ claims that the strong autocorrelation of $\delta O$ at $j=25$ (lower curve of fig. 3) is evidence for a new solar fluctuation. We feel that faculae provide an equally 


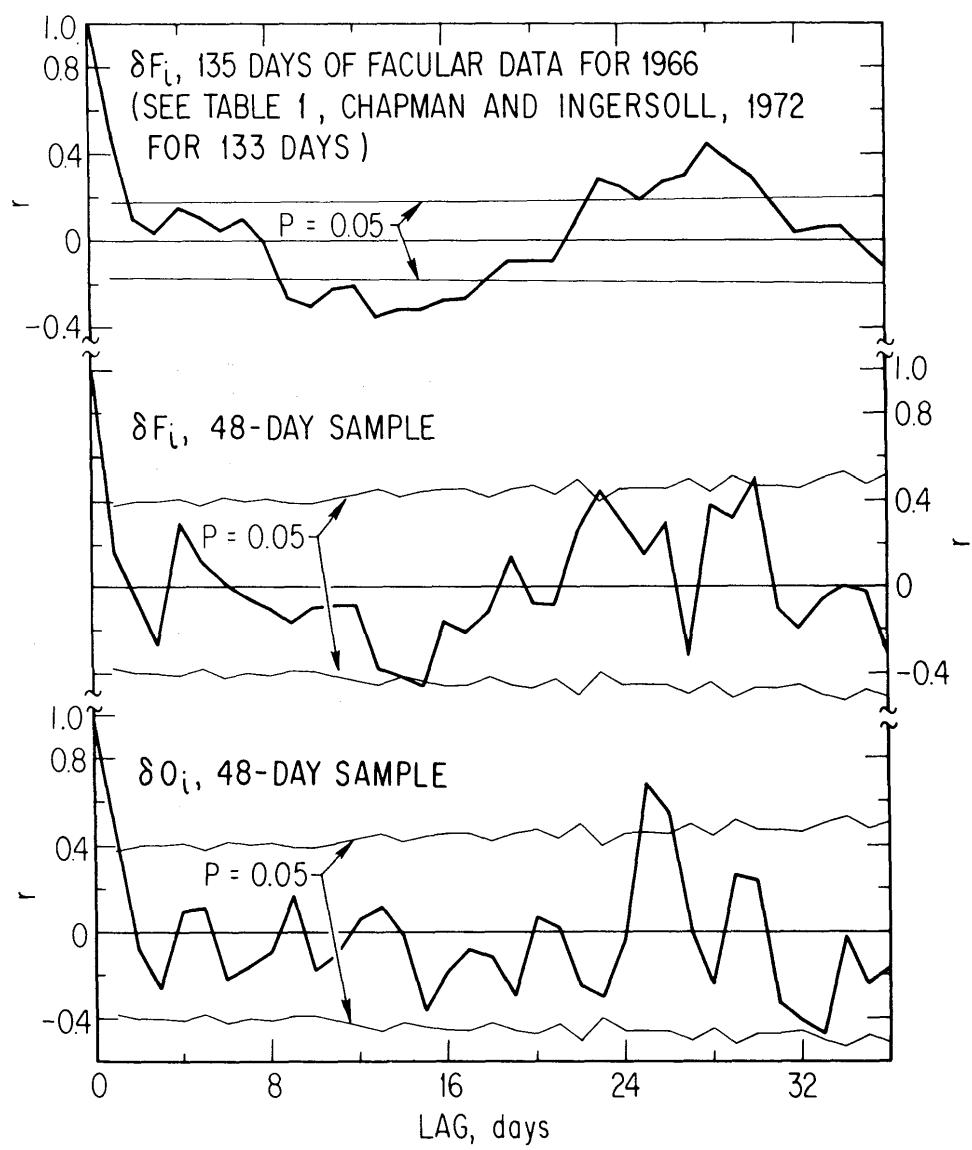

FIG. 3.-Lagged autocorrelations of (top to bottom) 135 facular residuals (133 from table 1 of Chapman and Ingersoll 1972), 48-day sample of facular residuals, and 48-day sample of oblateness residuals. The $P=0.05$ lines indicate the probability of a correlation coefficient from a random sample exceeding those values of the correlation coefficient. The solar rotation period is clearly evident in the topmost curve, but is less evident in the other curves. The double peak near $\frac{1}{2}$ solar rotation in the topmost curve is probably due to our measurement zones not being on a diameter.

attractive alternate explanation for this peak. The important point to keep in mind in intercomparing the three curves of figure 3 is that the differences are not statistically significant. For example, suppose that all three curves are samples from a parent population for which the correlation coefficient $r$ varies smoothly from 0.3 at $j=23$ to a maximum around 0.45 at $j=27$ down to 0.3 at $j=31$. At the 5 percent level, the only points which differ significantly from this parent population are the points $r=-0.32, j=27$ on the middle curve and $r=-0.24, j=28$ on the lower curve. Since 27 points are involved ( 9 points on each curve from $j=23$ to 31 ), a significant difference for two points is not surprising. The strong autocorrelation $(r \approx 0.7)$ of the oblateness data $\delta O$ at $j=25$ is not significant if one compares it with a parent population for which $r=0.4$. The number of data pairs $N_{j}=19$ for this lag is simply not sufficient to distinguish such differences at the 5 percent level.

Figure 4 presents autocorrelations for data from the 64-day sample, including the autocorrelation of $e_{i}$ defined in equation (6), for $\epsilon \rightarrow \infty$. Typical values of $N_{j}$, the number of data pairs, are now around 30. The general features of the curves for the 48-day sample are preserved. The low or negative correlation is still present in all curves around $j=27$. The large autocorrelation of $\delta O$ at $j=25$ is still present, but 


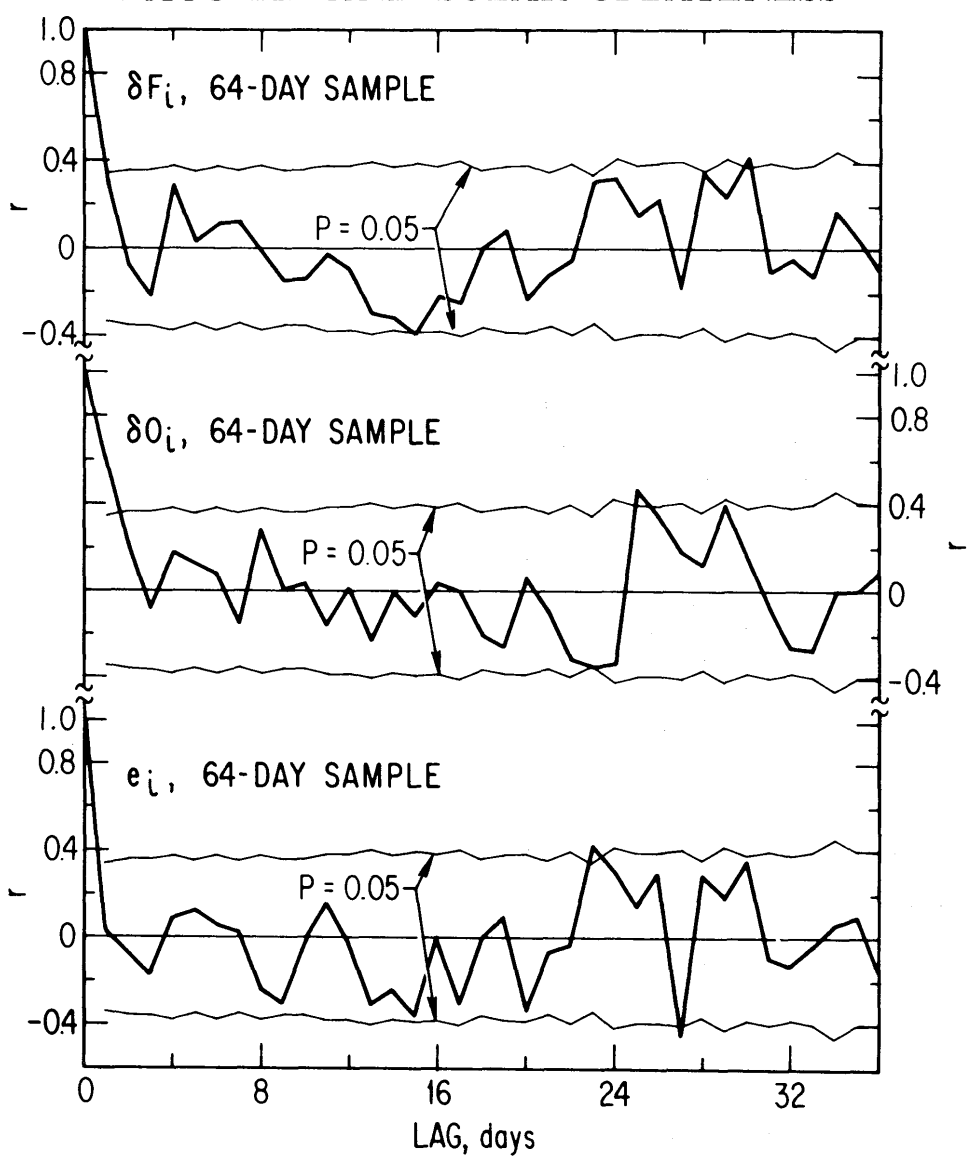

FIG. 4.-Lagged autocorrelations for the 64-day samples. The top curve is from the 64-day sample of facular residuals, the middle curve is from the 64-day sample of oblateness residuals, and the bottom curve is from the error defined by eq. (6) for the case $\epsilon=\infty$. (The curve for $\epsilon=0$ is not greatly different.)

now $r$ is about 0.45 instead of 0.67 . As discussed earlier, the autocorrelation at $j=1$ is significant for $\delta O$ and $\delta F$ but not for $e_{i}$. The autocorrelation of $e_{i}$ near 1 solar rotation is marginally significant, indicating that the autocorrelation which exists around 1 solar rotation has not been completely removed in our regression analysis for $\epsilon \rightarrow \infty$.

Figure 5 shows the cross-correlations of $\delta O$ and $\delta F$ lagged relative to each other up to two solar rotations. The oblateness data $\delta O_{i}$ were taken from the 64-day and 48-day samples, respectively. The facular data $\delta F_{i}$ were taken from 135 days, of which the first 133 days are given in table 1 of our earlier paper. (See Appendix B for additional 2 days.) The data have been folded so that data pairs $\left(\delta O_{i}, \delta F_{i+j}\right)$ and $\left(\delta O_{i}, \delta F_{i-j}\right)$ both contribute to the sums. Thus the number of data pairs $N_{j}$ is typically around $2 N$, where $N$ is either 64 or 48 . The number of data pairs at $j=0$ is just $N$. These numbers have been taken into account in computing the 0.05 levels of significance about the $r=0$ axes of the two correlations. We see that the strong cross-correlation ( $r=0.50$ for the 64-day sample, and $r=0.39$ for the 48-day sample) at $j=0$ falls off rapidly for $j>0$. When sunspots are included, $r(\delta O, \delta F)$ becomes 0.51 and 0.38 for the 64-day and 48-day samples, respectively. The dependence on solar rotation is easily discernible to the eye, and provides further evidence that $O$ and $F$ are statistically related. For the 64-day sample we find significant (probability $=P \leq 0.05$ ) correlations for $j=1 ; 10,11 ; 15,16 ; 27,28,29 ; 44,45 ; 54,55,56$. These can all best be 


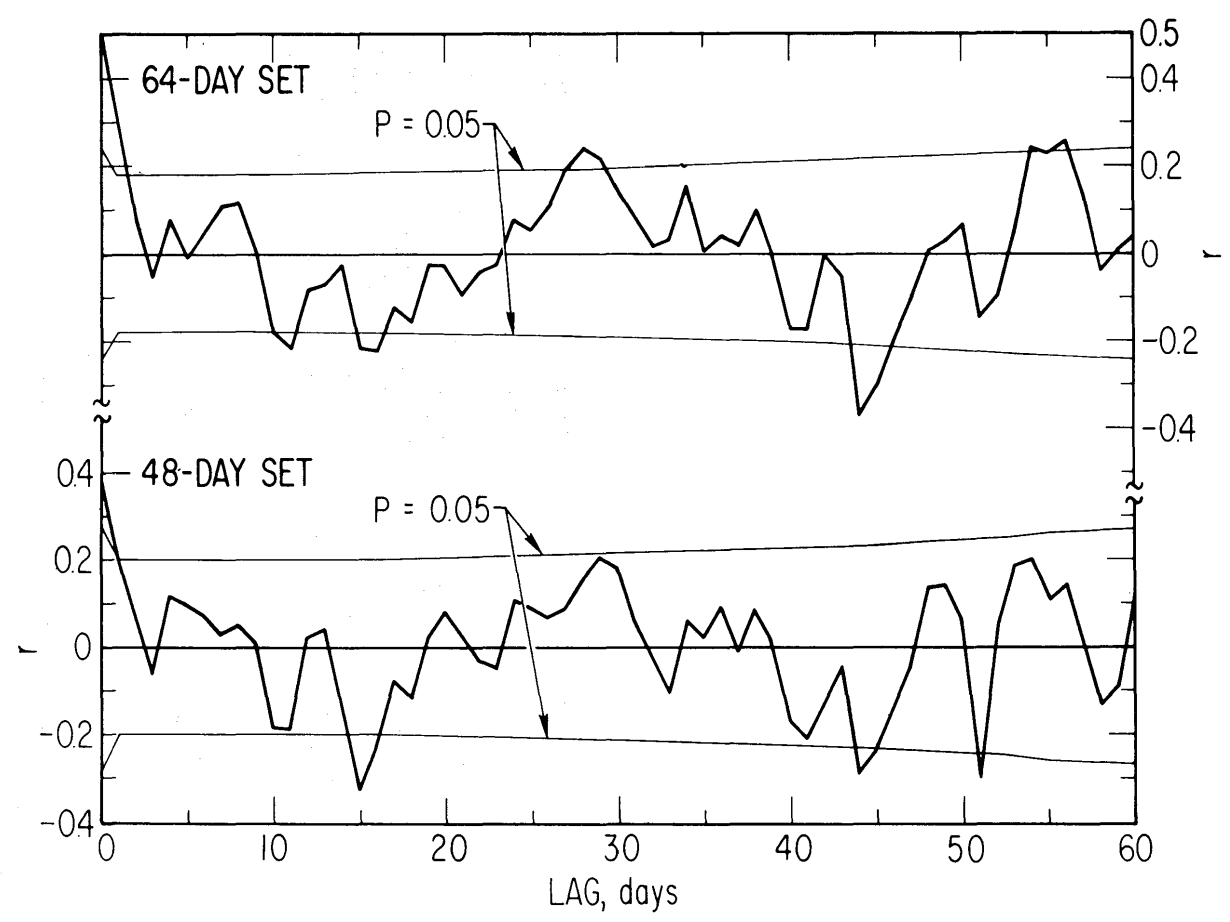

FIG. 5.-Lagged cross-correlations as defined by equation (7) for the 64-day and 48-day samples. The correlations have been folded about zero lag. The effect of solar rotation is clearly evident, especially for the 64-day sample. The effect of the measurement zones not being on a solar diameter is quite clear at $\frac{1}{2}$ rotation period as evidenced by the double negative peaks at 10-16 days lag.

explained by solar rotation. As explained before, the double peak at approximately $\frac{1}{2}$ solar rotation is a consequence of the measurement zones not being directly on a diameter. The small peak at $j=40$ and 41 is probably due to this same effect and is therefore probably real. All of these features are visible in the 48-day sample crosscorrelation, but fewer of them are statistically significant, partly because of the smaller sample size and partly because some of the correlation coefficients are numerically smaller. These values of $r(\delta O, \delta F)$ are smaller than the corresponding values of $r(\delta O, \delta O)$ and $r(\delta F, \delta F)$ shown in figures 3 and 4 . This difference could be due to systematic errors in the facular signal or to some other systematic difference between the two sets of data. For instance, the unfolded cross-correlations show a marked asymmetry about the origin $(j=0)$ that may be due to some mismatch of the two data sets. We do know that the facular signal does not refer to the same zone as that represented by Dicke's published data (Chapman and Ingersoll 1972, p. 828).

We have shown in this section that there exists a highly significant linear relationship between the facular signal $F$ and the oblateness signal $O$. Our estimate of the facular contribution to the excess solar oblateness ranges from about 31-35 percent to essentially 100 percent (for the 64-day sample, tables 1 and 2) depending on the choice of the error ratio $\epsilon$. This error ratio cannot be uniquely determined from the data $O_{i}, F_{i}$, and $\sin 2 P_{i}$ alone. In the next section we will make estimates of $\epsilon$ using additional information.

\section{DISCUSSION}

\section{a) Determination of Possible Values of $\epsilon$}

Probably the simplest determination of $\epsilon$ is obtained by combining the error flags in figure 1 of Dicke (1972a) into a single rms error for either the 64-day or the 48-day samples. This rms oblateness error can then be compared with the rms error of 
repeatability for the same 64-day or 48-day sample of facular data (Chapman and Ingersoll, p. 827). In units of $\sin 2 P$ the rms error of the oblateness data, $\sigma_{O}$, is 0.194 and 0.150 for the 64-day and 48-day samples, respectively. For the facular data the rms error of repeatability is 0.249 and 0.296 for the 64-day and 48-day samples, respectively. These numbers yield $\epsilon \geqslant 1.28$ for the 64-day sample and $\epsilon \geqslant 1.97$ for the 48-day sample, where the inequality expresses our feeling that the actual facular rms measurement error will be larger than the rms error of repeatability used here. These values of $\epsilon$ give facular contributions to the excess solar oblateness of about 50 percent for the 64-day sample and 22 percent for the 48-day sample. Including the effect of sunspots, these estimates become 61 and 35 percent, respectively. These minimum estimates of $\epsilon$ are indicated by vertical lines in figure 1 .

As noted in our earlier paper, the rms error of repeatability provides only a minimum estimate of facular error, and therefore only a minimum estimate of $\epsilon$. We believe there are sizable systematic (i.e., repeatable) errors in the facular signal, for several reasons. First, in deriving facular areas from the photographs, faculae could be detected only if their contrast were above some threshold value; yet the facular flux excess is the relevant quantity. Thus for pictures of low quality (out-of-focus, poor seeing, etc.) we will see only the larger groups of faculae, whereas a device that measures flux excess, such as the Princeton oblateness telescope, will not be so affected. Second, the two days that are required for regions to travel from our measurement zone to Dicke and Goldenberg's measurement zone can cause appreciable changes in the facular regions. Third, our measurement zone has a different size and shape from that of Dicke and Goldenberg (although their largest zone is similar to our measurement zone). These effects are known to exist and will tend to increase the effective value of $\epsilon$ (and thus of $C_{5}$ ), but the size of the increase is not known. Another possible systematic error in the facular signal might be a systematic or personal error in estimating facular areas by eye. Whether this error is present to a significant degree is not known.

Another estimate of $\epsilon$ is the ratio of the residuals from $\sin 2 P$ of the two sets of signals. Very recently, Dicke $(1972 c)$ has concluded that most (84 percent) of the mean square residual of $O$ from $\sin 2 P$ is due to solar phenomena rather than to errors of measurement. Dicke feels there is considerable evidence against bright or dark spots on the Sun (e.g., faculae or sunspots) as sources of this fluctuation in his signal, and that their source is probably gravitational. If Dicke is correct, then most of the rms residual of $O$ from $\sin 2 P$ is due to solar phenomena unrelated to faculae, and must be classified as "error" in the present analysis. This leads to an estimate of $\epsilon$ which is the ratio of the rms residual of $F$ from $\sin 2 P$ to the rms residual of $O$ from $\sin 2 P$. For the 64-day sample this ratio is 1.61 , and for the 48-day sample it is 2.77 . From figure 1 , the corresponding estimates of the facular contribution to the excess oblateness signal are 63 and 36 percent (74 and 60 percent including sunspots), respectively. On the other hand, if the solar-related part ( 84 percent) of the mean square residual of $O$ from $\sin 2 P$ were due to faculae, then the error of $O$ would be much smaller, and we would have $\epsilon \approx 5$ for the 64-day sample, and $\epsilon \approx 10$ for the 48-day sample. In this case, the corresponding estimates of the facular contribution to the excess oblateness signal are essentially 100 percent. Thus, although we cannot estimate $\epsilon$ with precision, we feel there is little reason to reject the possibility that faculae contribute an important part of the excess oblateness signal.

\section{b) Comparison of the 64-Day and 48-Day Samples}

We now compare the results obtained for the 64-day sample with those obtained for the 48-day sample. For the 64-day sample, the correlation coefficient $r$ is larger, and the ratio of the value of $C_{5}$ to its standard deviation is greater by a factor of 1.6 , compared to the 48-day sample. These results imply that the degree of correlation between $O$ and $F$ is greater for the 64-day sample than for the 48-day sample. Dicke 
(1972a) considers the oblateness data less reliable for the 64-day sample because of additional measurement errors associated with 16 days at the beginning and end of the 1966 observing season. Indeed, we find, based on the error bars in figure 1 of Dicke (1972a), that these 16 days have an rms error about 40 percent larger than that for the 48-day sample. This increased error partly explains the smaller value of $\epsilon$ obtained for the 64-day sample compared to the 48-day sample. However, since there is little reason to expect measurement errors of $O$ to be related to residuals of $F$, we conclude that the additional measurement errors of $O$ associated with the less reliable days are small compared to the residuals of $O$ associated with faculae. In other words, for the purposes of this discussion, there appears to be no great difference between the 16 less reliable days and the 48 remaining days in the total 64-day sample. Thus we favor the results based on the 64-day sample because it contains more information.

\section{DISCUSSION OF DICKE (1972a)}

\section{a) Discussion of Dicke's Figure 1}

We first discuss figure 1 of Dicke's rebuttal paper. Dicke divided the oblateness signal into three nearly equal classes according to the absolute value $\left|F_{i}\right|$ of the facular signal in table 1 of our earlier paper. He then regressed the oblateness signal against $\sin 2 P$ for each class and found no significant difference in the regression coefficients for the three classes (see fig. 1 of Dicke 1972a for the regression coefficients). Since Dicke found that the value of $\langle|F|\rangle$ for the three groups varied by over a factor of 10 , he concluded that there was no evidence for an important contribution from faculae to the solar oblateness signal.

Dicke's approach is roughly equivalent to a three-point regression analysis in which the independent variable is the mean value of $\left|F_{i}\right|$ for each of the three classes, and the dependent variable is the mean oblateness signal as measured by his regression coefficient for each of the three classes. However, this choice of independent and dependent variables is valid only when $\epsilon \rightarrow 0$, that is, when the error in $\langle|F|\rangle$ is so small that the number of incorrect assignments of days is negligible. In fact, we find that for the topmost group (smallest $\langle|F|\rangle$ ) the rms error of repeatability is 0.15 , which is larger than $\langle|F|\rangle$ itself. More importantly, we have shown that $\epsilon>1$, and possibly $\epsilon \gg 1$. For the case $\epsilon \rightarrow \infty$, the variables $O$ and $F$ should exchange roles. The reasoning is the same as that following equations (A7) and (A8) in Appendix A of this paper. Thus for $\epsilon \rightarrow \infty$ it is more appropriate to use the excess oblateness $\left|O_{i}-0.185 \sin 2 P_{i}\right|$ to divide the days into three classes and to let the mean facular signal (as measured by the coefficient $C_{2}$ appearing in eq. [1]) be the dependent variable. This is the approach taken in figure 6 of this paper.

The top three curves of figure 6 show the facular signal $F_{i}$ for 64 days of observation divided into three classes according to the absolute value of the excess oblateness signal. The data $F_{i}$ in each class have been fitted separately to a curve of the form

$$
F=C_{2} \sin 2 P+C_{4} .
$$

The approach is essentially equivalent to that in figure 1 of Dicke (1972a), except that the roles of $O$ and $F$ are reversed. For the three classes, characterized by low, medium, and high values of the excess oblateness signal, the values of $C_{2}$ are $0.19 \pm 0.39$, $0.90 \pm 0.43$, and $1.53 \pm 0.51$, respectively. For the 48-day sample the corresponding values of $C_{2}$ are $1.41 \pm 1.0,0.16 \pm 0.96$, and $1.85 \pm 0.57$, respectively, for low, medium, and high values of the excess oblateness signal. From these numbers we conclude that although there is considerable scatter in the values of $F$, there is a significant trend as evidenced by the increasing values of $C_{2}$ for the three classes. We 


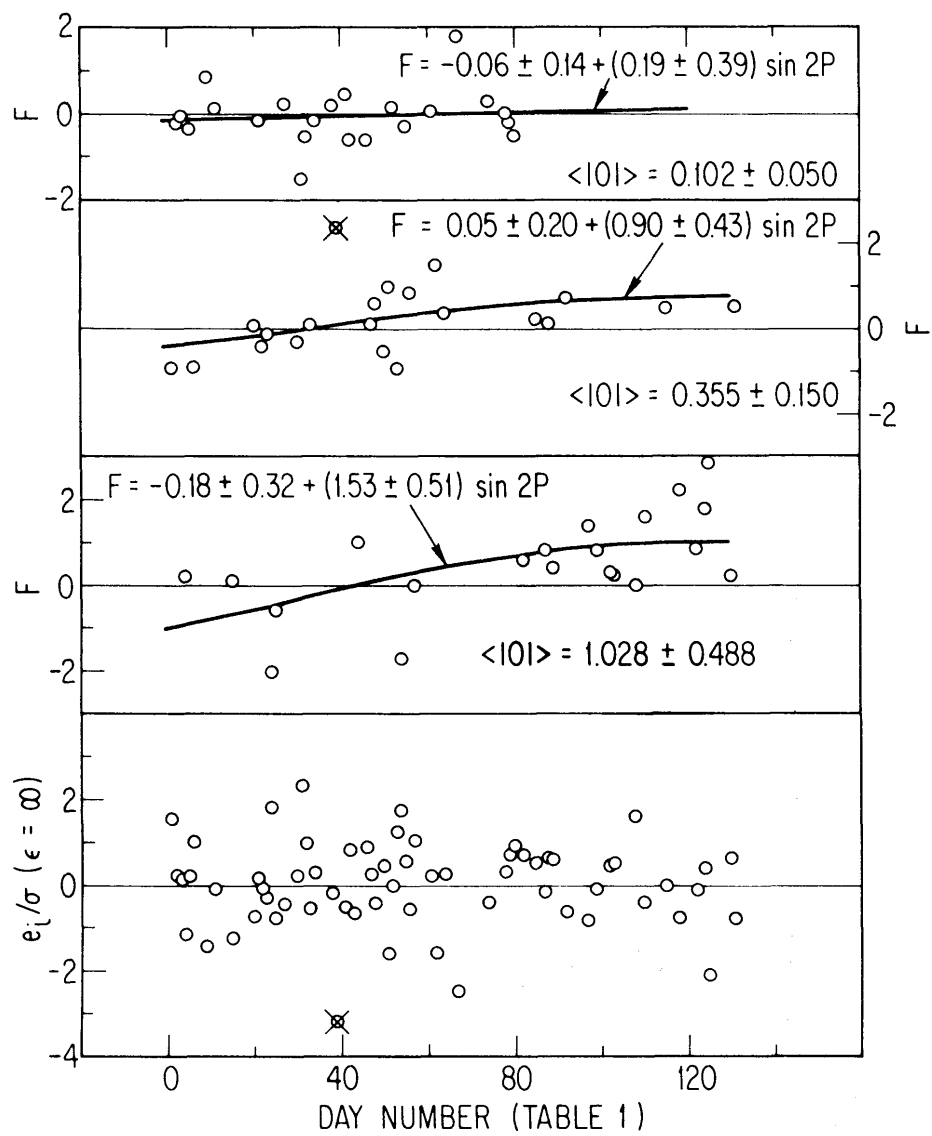

FIG. 6.-Upper three curves: Same as fig. 1 of Dicke (1972a) but with the roles of $O$ and $F$ reversed. This reversal, in which the excess oblateness signal rather than the facular signal is treated as the independent variable, is correct if the facular error is larger than the oblateness error. Notice that the dependence of $F$ on $\sin 2 P$ increases as the mean excess oblateness, $\langle|O|\rangle$, increases. This trend indicates that a significant relationship exists between $O$ and $F$. Bottom curve, errors, $e_{i} / \sigma$, defined by equation (6) versus day number, $i$. The points with an $x$ are from day 39 which has an unusually large sunspot signal.

offer this analysis to counter figure 1 of Dicke (1972a) and to show how the results depend on the value of $\epsilon$ assumed.

We shall continue to base our conclusions on the results of the complete regression analysis, the results of which are presented in figure 1 and tables 1 and 2 of this paper. We emphasize again that there is no way to infer the correct value of $\epsilon$ from the data $O_{i}, F_{i}$, and $\sin 2 P_{i}$ alone. Figure 1 of Dicke (1972a) assumes $\epsilon=0$ whereas we have shown $\epsilon$ to be at least as great as 1.6 and probably much larger. Errors in the independent variable (which is $F$ in Dicke's fig. 1) not only reduce the degree of correlation but also decrease the regression coefficient (Ezekiel and Fox 1959). Perhaps the oblateness signal should be the independent variable as in our figure 6.

One obvious feature of Dicke's figure 1 is the apparent occurrence of oblateness signals with small errors in the upper part of the figure. This clustering does not seem to be statistically significant at the 25 percent level in a linear regression analysis against either $|\delta F|$ or $|\delta O|$. However, since many faculae do occur at the limbs in patches, they might cause guiding and seeing errors to result in an unusually noisy signal. Although Dicke has rejected only 5 percent of his data (Dicke 1972a, p. 832), 
those rejected data may tend to be from those days with large facular areas present on the limb more often than by chance.

\section{b) The "Non-Gaussian Character of F"}

As Dicke pointed out on page 833 of his rebuttal paper (Dicke 1972a), one should subtract a "seasonal trend" (i.e., $\sin 2 P$ ) from both the oblateness and the facular signals before calculating correlation coefficients. Dicke did not do so for the facular data because of the "non-Gaussian character of $F$." For the data of table 1 of our first paper the fit of $F$ to $\sin 2 P$ is $6 \sigma$ significant. For the 64-day set and the 48-day set the fit to $\sin 2 P$ is $5 \sigma$ and $2.5 \sigma$ significant, respectively. It is not justified statistically to neglect this strong seasonal trend in $F$. Neglect of this trend causes an underestimate in the corresponding regression coefficients. Figure 7 shows the distribution of $\delta F$, including the effect of sunspots, for the 64-day sample. Both this distribution and that for the 48-day sample are reasonably Gaussian. The moments of the distribution are $\mu_{3} / 3 \sigma^{3}=0.013$ and $\left(\mu_{4} / 3 \sigma^{4}\right)-1=0.25$ for the 64-day sample, and -0.138 and 0.164 for the 48 -day sample, respectively. These same quantities for the oblateness data are 0.129 and 0.354 for the 64-day sample and -0.142 and +0.017 for the 48-day sample, respectively.

However, of greater importance is the fact that we require neither $F$ nor $\delta F$ but only the errors $e_{i}$ in our regression equation (4) to be Gaussian. Figure 2 shows that these errors are Gaussian. Furthermore, one would not necessarily expect $\delta O, \delta F$, or any natural phenomenon to behave in a Gaussian fashion.

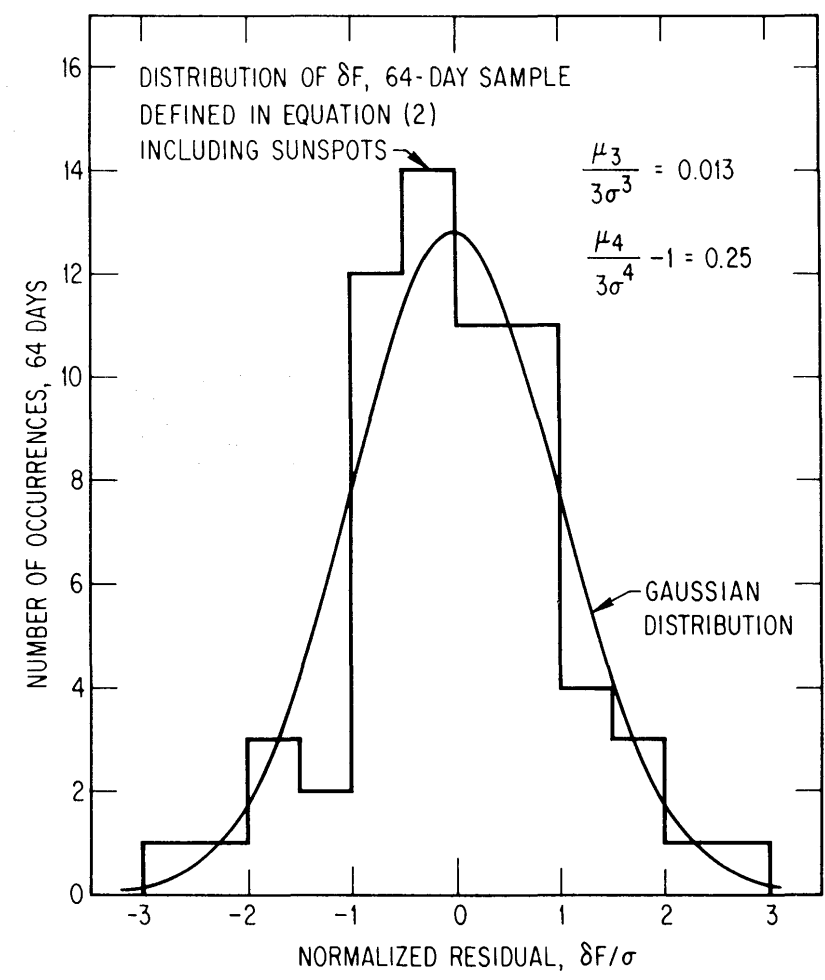

FIG. 7.-Distribution of residuals of the facular signal defined in eq. (2), including the effect of sunspots, for the 64-day sample. The distribution for the 48-day sample, with sunspots, is not greatly different but is slightly more skewed. 


\section{c) Additional Comments}

We now comment briefly on some points raised in our earlier paper which were discussed recently by Dicke (1973). We showed that faculae could cause a $\delta^{1 / 2}$ dependence of the observed oblateness, and such a dependence appears to be consistent with the Princeton data. This $\delta$-dependence of the data was first explicitly noted by Ingersoll and Spiegel (1971), and is confirmed by Dicke (1973). For the three magnifications, $\delta=6.5,12^{\prime \prime} .9$, and 19.1 , he gives $\Delta r_{d}=0.040 \pm 0.006$, $0.044 \pm 0.006$, and $0.050 \pm 0.007$, respectively, where $\Delta r_{d}$ is the inferred radius excess at the solar equator. The relative brightness $B$ of the photosphere at the edge of the occulting disk is approximately $0.360,0.400$, and 0.432 , respectively (Dicke $1970 a$ ), which yields $B \Delta r_{d}=00^{\prime \prime} 0144,0.0176$, and 0.0216 , for each of the three magnifications. The latter three numbers are proportional to the observed equatorial flux excess, and are approximately proportional to $\delta^{1 / 2}$. We obtain the same $\delta$-dependence using $\left\langle a_{m}+b_{m}\right\rangle,\left\langle a_{m}\right\rangle$, and $\left\langle a_{m}-b_{m}\right\rangle$ from Dicke $(1972 c)$ as estimates of $\Delta r_{d} / r$ for the three values of $\delta$, where the averages are taken either for the 64-day or for the 48-day data set.

We now comment on Dicke's (1973) use of our facular function $F$ to correct his data. Apparently he fits one set of $F_{i}$ to each of the three sets of $\delta O_{i}$, corresponding to the three magnifications, and then subtracts off the fitted function to obtain the corrected oblateness residuals for each magnification. The fitting is done with $F$ rather than $\delta F$, and with the implicit assumption $\epsilon=0$. We have already given our criticisms of these two procedures. Another criticism arises from the fact that our facular function $F$ refers only to a single zone, and this corresponds most closely to Dicke's largest magnification (largest $\delta$ ). Thus one would expect the fit between $\delta O$ and $F$ or between $\delta O$ and $\delta F$ to improve with increasing $\delta$. We do not know the $O_{i}$ for each magnification separately, but as an estimate we may use $a_{m}+b_{m}, a_{m}$, and $a_{m}-b_{m}$ from Dicke $(1972 c)$. For the 64-day sample, the correlation coefficients of these three quantities with $\delta F$ are $0.18,0.50$, and 0.57 , respectively, which shows the expected increase with $\delta$. This effect accounts for at least some of the increase of Dicke's facular corrections with $\delta$, and therefore explains, at least partially, why the corrected oblateness signals in Dicke's (1973) figures show so little $\delta$-dependence: the facular corrections for the larger magnifications are too large relative to those for the smaller magnifications.

Finally, we remind the reader that our earlier paper contained an independent estimate of the facular contribution to Dicke and Goldenberg's signal. This estimate relied on a simple facular model to give facular limb darkening in the poorly observed limb region. The model fitted known observations (Chapman 1970; Rogerson 1961) reasonably well from the center of the disk to within $5^{\prime \prime}$ from the limb, at which point foreshortening becomes so severe that available observations are not of much help. Measurements of facular contrast near the limb are much degraded by seeing and telescope resolution. One needs observations of facular flux excess, not contrast, as a function of limb position. There is no evidence to show that our model predictions of facular flux excess are seriously in error in the important region from $1^{\prime \prime}$ to $20^{\prime \prime}$ from the limb. This model, together with Allen's (1963) value for $\bar{A}$, the mean area of faculae on the solar disk, gave an estimate of the facular signal which was essentially equal to the excess observed oblateness signal.

We also get the same result using our own estimate of $\bar{A}$. Our value of $\bar{A}$ obtained from photographs is $4.6 \times 10^{-3}$, which is 2.7 times larger than Allen's value. However, the contrast of faculae near the $\operatorname{limb}(\cos \theta \approx 0.1)$ in these photographs is about 0.20 , which is 2.5 times smaller than in figure 1 of our first paper. These two factors nearly cancel, leaving the product essentially the same as before ( 8 percent larger). The use of Rogerson's observations were important for determining the shape of the 
facular limb darkening since the foreshortening near the limb of faculae will cause an excessively rapid falloff in facular contrast for most ground-based observations. This excessively rapid falloff would then cause one to underestimate the facular flux excess near the limb.

\section{SUMMARY}

We have shown that faculae have contributed some of Dicke and Goldenberg's excess oblateness signal. Objective (and we feel conservative) estimates are 61 percent for the 64-day sample $(\epsilon=1.28)$ and 35 percent for the 48-day sample $(\epsilon=1.97)$. These estimates correspond to the vertical lines in figure 1. The main point of our original paper is still valid, that faculae may have contributed all of Dicke and Goldenberg's excess oblateness signal. When Dicke and Goldenberg release all of their measurements, particularly for their $19^{\prime \prime}$ zone, our hypothesis can be more fully tested.

We appreciate helpful criticisms of the first draft of this paper by an unknown referee. We gratefully acknowledge support of this research by company funds of The Aerospace Corporation and by the National Aeronautics and Space Administration under grant NGL 05-002-003 awarded to the California Institute of Technology. We thank Dr. Eldon L. Haines for bringing to our attention the paper by Chandler (1972). We also thank Dr. Dicke for sending some of his data to us (Dicke 1972b).

\section{APPENDIX A}

As described in $\S$ II, we wish to fit a linear equation to the data $O_{i}, F_{i}$, and $\sin 2 P_{i}$, given that both $O$ and $F$ are subject to error. The observed values $O_{i}$ and $F_{i}$ differ from the "true" values by unknown amounts. The variances are $\sigma_{O}{ }^{2}$ and $\sigma_{F}{ }^{2}$, and the covariance is zero. We use a generalized method of least squares described by Deming (1938) and more recently by Chandler (1972).

Following these authors, we define the "adjusted" values $\hat{O}_{i}$ and $\hat{F}_{i}$ as our estimates of the true values of $O$ and $F$ for the $i$ th day of observation. The adjusted values are constrained to satisfy the linear equation

$$
\hat{O}_{i}=C_{5} \hat{F}_{i}+C_{6} \sin 2 P_{i}+C_{7} .
$$

We determine $\hat{O}_{i}, \hat{F}_{i}$, and the coefficients $C_{5}, C_{6}, C_{7}$ by minimizing $\chi^{2}$, where

$$
\chi^{2}=\sum_{i}\left[\left(\frac{O_{i}-\hat{O}_{i}}{\sigma_{O}}\right)^{2}+\left(\frac{F_{i}-\hat{F}_{i}}{\sigma_{F}}\right)^{2}\right] .
$$

We first substitute for $\hat{O}_{i}$ using equation (A1) and set

$$
\partial \chi^{2} / \partial \hat{F}_{i}=0, \quad \text { all } i
$$

Each $\hat{F}_{i}$ appears only once, and we obtain

$$
\hat{F}_{i}=\frac{\sigma_{O}{ }^{2} F_{i}+C_{5} \sigma_{F}{ }^{2}\left(O_{i}-C_{6} \sin 2 P_{i}-C_{7}\right)}{\sigma_{O}{ }^{2}+C_{5}{ }^{2} \sigma_{F}{ }^{2}} .
$$

This is substituted into equation (A2), which yields

$$
\chi^{2}=\sum_{i}\left[\frac{\left(O_{i}-C_{5} F_{i}-C_{6} \sin 2 P_{i}-C_{7}\right)^{2}}{\sigma_{O}{ }^{2}+C_{5}{ }^{2} \sigma_{F}{ }^{2}}\right] .
$$


Minimizing with respect to $C_{6}$ and $C_{7}$ yields

$$
C_{6}=1-C_{5}, \quad C_{7}=C_{3}-C_{4} C_{5},
$$

since $O$ and $F$ are scaled to $\sin 2 P$ as described in $\S$ II, equation (1). The expression for $\chi^{2}$ may now be rewritten in terms of residuals from $\sin 2 P$, defined in $\S \mathrm{II}$, equation (2):

$$
\chi^{2}=\sum_{i}\left[\frac{\left(\delta O_{i}-C_{5} \delta F_{i}\right)^{2}}{\sigma_{O}{ }^{2}+C_{5}{ }^{2} \sigma_{F}^{2}}\right]
$$

Minimizing this expression then yields $C_{5}$ as a function of the error ratio $\epsilon=\sigma_{F} / \sigma_{O}$.

These expressions assume a more familiar form in the limits $\epsilon \rightarrow 0$ and $\epsilon \rightarrow \infty$. In the limit $\epsilon \rightarrow 0$, minimizing $\chi^{2}$ in equation (A4) or (A6) is equivalent to minimizing $\sigma_{O}^{2} \chi^{2}$, which is given by

$$
\sigma_{O}^{2} \chi^{2}=\sum_{i}\left(O_{i}-C_{5} F_{i}-C_{6} \sin 2 P_{i}-C_{7}\right)^{2}=\sum_{i}\left(\delta O_{i}-C_{5} \delta F_{i}\right)^{2}
$$

In the limit $\epsilon \rightarrow \infty$, minimizing $\chi^{2}$ is equivalent to minimizing ${\sigma_{F}}^{2} \chi^{2}$, which is given by

$$
\sigma_{F}^{2} \chi^{2}=\sum_{i}\left(F_{i}-\frac{1}{C_{5}} O_{i}+\frac{C_{6}}{C_{5}} \sin 2 P_{i}+\frac{C_{7}}{C_{5}}\right)^{2}=\sum_{i}\left(\delta F_{i}-\frac{1}{C_{5}} \delta O_{i}\right)^{2} .
$$

Thus when the error of one variable is much greater than that of the other variable, our method reduces to the more familiar method of least squares, with the variable having the larger error assuming the role of dependent variable. In his analysis of these data, Dicke $(1972 a)$ regresses $\delta O$ against $F$, with $\delta O$ as dependent variable. This is roughly equivalent to equation (A7), although Dicke uses the full value of the facular signal $F$, rather than $\delta F$ as we have done here. It can be shown, however, that the value of $C_{5}$ obtained from equation (A7), corresponding to the case $\epsilon \rightarrow 0$, is simply a lower-bound estimate of the true value. The upper-bound estimate is obtained from equation (A8), corresponding to $\epsilon \rightarrow \infty$. This difference of interpretation is perhaps the greatest difference between our work and Dicke's.

For any given $\epsilon$, the values of $\sigma_{O}$ and $\sigma_{F}$ may be estimated directly from the data. We take

$$
\chi^{2}=(N-1),
$$

where $N$ is the number of days of observation in the sample. The above relation follows from the definition of $\chi^{2}$ (eq. [A4] or [A6]) and the fact that errors of $O$ and errors of $F$ are uncorrelated. Thus the ensemble mean of each bracketed term in equation (A4) or (A6) is equal to unity, from which it follows that an estimate of $\chi^{2}$ from one realization of the data is equal to the number of degrees of freedom $(N-1)$. Equations (A6) and (A9) are sufficient to estimate $\sigma_{O}{ }^{2}$ and $\sigma_{F}{ }^{2}$ for any value of $\epsilon$.

With estimates of ${\sigma_{O}}^{2}$ and ${\sigma_{F}}^{2}$, we can estimate the variance $\sigma_{5}{ }^{2}$ of the coefficient $C_{5}$. Following Bevington (1969, p. 245), we take

$$
\sigma_{5}^{2}=\left[\frac{1}{2} \frac{\partial^{2} \chi^{2}}{\partial C_{5}^{2}}\right]^{-1}
$$

An important quantity is $C_{5} / \sigma_{5}$, which measures the statistical significance of our results. Using equations (A6), (A9), and (A10) we find

$$
\frac{C_{5}}{\sigma_{5}}=\left[\frac{(N-1) \alpha_{O F} C_{5}}{\alpha_{O O}-2 C_{5} \alpha_{O F}+C_{5}^{2} \alpha_{F F}}\right]^{1 / 2},
$$


where we have defined

$$
\alpha_{O O}=\sum_{i}\left(\delta O_{i}\right)^{2}, \quad \alpha_{O F}=\sum_{i}\left(\delta O_{i}\right)\left(\delta F_{i}\right), \quad \alpha_{F F}=\sum_{i}\left(\delta F_{i}\right)^{2} .
$$

Expression (A11) for $C_{5} / \sigma_{5}$ depends on $\epsilon$ through the coefficient $C_{5}$. The maximum value of $C_{5} / \sigma_{5}$ occurs at $\epsilon^{2}=1 / C_{5}{ }^{2}=\alpha_{F F} / \alpha_{O O}$, and is given by

$$
\left(\frac{C_{5}}{\sigma_{5}}\right)_{\max }=\left[\frac{(N-1) r}{2(1-r)}\right]^{1 / 2}, \quad \epsilon^{2}=\alpha_{F F} / \alpha_{O O} .
$$

Here we have defined $r$, the correlation coefficient of residuals, as follows:

$$
r=\alpha_{O F}\left(\alpha_{O O} \alpha_{F F}\right)^{-1 / 2} .
$$

The estimate (A13), obtained by setting $C_{5}$ equal to the ratio of rms residuals $\left(\alpha_{O O} / \alpha_{F F}\right)^{1 / 2}$, was given in our earlier paper as one possible interpretation of the data. Now we see that this estimate is not unique, because $\epsilon$ is not known a priori. On the other hand, it does provide the sharpest fit in the sense that the curve $\chi^{2}$ versus $C_{5}$ has a narrower minimum at this value of $C_{5}$ than at any other value, according to the definition (A10).

The minimum value of $C_{5} / \sigma_{5}$ occurs twice, at $\epsilon \rightarrow 0$ and at $\epsilon \rightarrow \infty$. This minimum value is given by

$$
\left(\frac{C_{5}}{\sigma_{5}}\right)_{\min }=\left[\frac{(N-1) r^{2}}{1-r^{2}}\right]^{1 / 2}, \quad \epsilon \rightarrow 0, \epsilon \rightarrow \infty,
$$

where $r$ has the same meaning as in equation (A13). Because it is a minimum value, equation (A15) provides a conservative estimate of $C_{5} / \sigma_{5}$. We use this equation in testing for statistical significance of the coefficient $C_{5}$.

In testing for statistical significance, we assume that errors of $O$ and $F$ are Gaussian and that errors from day to day are independent. These assumptions may be tested for any $\epsilon$. The adjusted values $\hat{O}_{i}$ and $\hat{F}_{i}$ are our estimates of the true values. Thus our estimates of the errors may be obtained from equations (A1) and (A3), and are given by

$$
\begin{aligned}
& \left(F_{i}-\hat{F}_{i}\right)=\left(\frac{C_{5}{ }^{2} \sigma_{F}{ }^{2}}{\sigma_{O}{ }^{2}+C_{5}{ }^{2} \sigma_{F}{ }^{2}}\right)\left(F_{i}-\frac{1}{C_{5}} O_{i}+\frac{C_{6}}{C_{5}} \sin 2 P_{i}+\frac{C_{7}}{C_{5}}\right), \\
& \left(O_{i}-O_{i}\right)=\left(\frac{\sigma_{O}{ }^{2}}{\sigma_{O}{ }^{2}+C_{5}{ }^{2} \sigma_{F}{ }^{2}}\right)\left(O_{i}-C_{5} F_{i}-C_{6} \sin 2 P_{\imath}-C_{7}\right) .
\end{aligned}
$$

Notice that both these estimates are proportional to the quantity

$$
e_{i}=O_{i}-C_{5} F_{i}-C_{6} \sin 2 P_{i}-C_{7},
$$

which we shall call simply the "error" in equation (A1). Thus to make a valid test of statistical significance, it is necessary that the $e_{i}$ have a Gaussian distribution and that the $e_{i}$ on different days be uncorrelated. We test these hypotheses in $\S$ II, using the $e_{i}$ obtained from the data $O_{i}, F_{i}$, and $\sin 2 P_{i}$.

\section{APPENDIX B}

Table B1 gives the sunspot signal for every day having noticeable sunspots during the time covered by table 1 of Chapman and Ingersoll (1972). These numbers were 
TABLE B1

1966 SUNSPOT SignALS

\begin{tabular}{cc|cc|cc}
\hline \hline $\begin{array}{c}\text { Day } \\
\text { No. }\end{array}$ & $\begin{array}{c}\text { Sunspot } \\
\text { Signal }\end{array}$ & $\begin{array}{l}\text { Day } \\
\text { No. }\end{array}$ & $\begin{array}{c}\text { Sunspot } \\
\text { Signal }\end{array}$ & $\begin{array}{c}\text { Day } \\
\text { No. }\end{array}$ & $\begin{array}{c}\text { Sunspot } \\
\text { Signal }\end{array}$ \\
\hline $1 \ldots \ldots$ & -0.0674 & $50 \ldots \ldots$ & -0.3954 & $104 \ldots \ldots$ & 0.0039 \\
$2 \ldots \ldots$ & -0.0667 & $54 \ldots \ldots$ & -0.1075 & $105 \ldots \ldots$ & 0.4709 \\
$6 \ldots \ldots$ & -0.1319 & $55 \ldots \ldots$ & +0.0667 & $110 \ldots \ldots$ & 0.2800 \\
$16 \ldots \ldots$ & -0.1301 & $56 \ldots \ldots$ & -0.1403 & $112 \ldots \ldots$ & 0.0244 \\
$24 \ldots \ldots$ & -0.0451 & $61 \ldots \ldots$ & +0.0700 & $114 \ldots \ldots$ & 0.1400 \\
$25 \ldots \ldots$ & -0.0686 & $63 \ldots \ldots$ & +0.4488 & $117 \ldots \ldots$ & 1.5386 \\
$28 \ldots \ldots$ & +0.1010 & $67 \ldots \ldots$ & +0.2100 & $119 \ldots \ldots$ & 0.2779 \\
$30 \ldots \ldots$ & -0.0538 & $78 \ldots \ldots$ & +0.0015 & $124 \ldots \ldots$ & 0.1890 \\
$31 \ldots \ldots$ & -0.0538 & $83 \ldots \ldots$ & -0.0323 & $125 \ldots \ldots$ & 0.0698 \\
$39 \ldots \ldots$ & +1.9232 & $85 \ldots \ldots$ & -0.0020 & $126 \ldots \ldots$ & 0.0039 \\
$40 \ldots \ldots$ & +0.6506 & $90 \ldots \ldots$ & +1.0219 & $128 \ldots \ldots$ & 0.0193 \\
$42 \ldots \ldots$ & -0.0871 & $95 \ldots \ldots$ & +0.0294 & $132 \ldots \ldots$ & 1.0725 \\
$43 \ldots \ldots$ & +0.1010 & $96 \ldots \ldots$ & -0.0294 & $133 \ldots \ldots$ & 0.0273 \\
$45 \ldots \ldots$ & +0.1106 & $97 \ldots \ldots$ & 0.0000 & & \\
\hline
\end{tabular}

Note.-Day no. 1 corresponds to 1966 June 1 as was the case in table 1 of Chapman and Ingersoll (1972).

obtained in a way similar to that outlined in $\S$ IV of our earlier paper. The area and latitude of the sunspots were determined by eye from photographs obtained at the San Fernando Observatory. The zones were the same as those used for measuring the facular signal. The areas and latitudes were then substituted into equation (10) of our earlier paper. The numbers so obtained were then scaled by the same factor, $f$, used to scale the facular signal to $\sin 2 P$. To modify the facular signal $F$, for each day, one simply subtracts the number in table $\mathrm{B} 1$ from the corresponding number appearing in table 1 of our earlier paper. The result of the scaling is equivalent to specifying that the mean sunspot signal is -4.2 percent of the mean facular signal, assuming that there is no systematic difference between the way the eye measures sunspot areas and facular areas. Days not listed should be considered to have no sunspot signal.

Two additional days having a measured facular signal and not included in table 1 of Chapman and Ingersoll (1972) are presented here. They are day numbers 132 and 133 (1966 October 10 and 11), for which the corresponding signals are 1.52 and 0.52 , respectively.

\section{REFERENCES}

Allen, C. W. 1963, Astrophysical Quantities (2d ed.; London: Athlone Press), p. 167.

Bevington, P. R. 1969, Data Reduction and Error Analysis for the Physical Sciences (New York: McGraw-Hill).

Chandler, J. P. 1972, Technometrics, 14, 71.

Chapman, G. A. 1970, Solar Phys., 14, 315.

Chapman, G. A., and Ingersoll, A. P. 1972, Ap. J., 175, 819.

Deming, W. E. 1938, Statistical Adjustment of Data (New York: Wiley 1943; Dover 1964).

Dicke, R. H. 1970a, Ap. J., 159, 1.

- 1970b, Ann. Rev. Astr. and Ap., 8, 297.

- $1972 a$, Ap.J., 175, 831.

- $1972 b$, private communication.

—_. 1972c, Ap. J., 176, 479.

1973, Solar Oblateness and Equatorial Brightening, Ap. J., 180, 293.

Dicke, R. H., and Goldenberg, H. M. 1967, Phys. Rev. Letters, 18, 313.

Ezekial, M., and Fox, K. A. 1959, Methods of Correlation and Regression Analysis: Linear and Curvilinear (New York: Wiley).

Ingersoll, A. P., and Spiegel, E. A. 1971, Ap. J., 163, 375.

Rogerson, J. B. 1961, Ap.J., 134, 331. 
